\title{
Vision Modulates Corticospinal Suppression in a Functionally Specific Manner during Movement of the Opposite Limb
}

\author{
Richard G. Carson ${ }^{1,2}$ and Kathy L. Ruddy ${ }^{1}$ \\ ${ }^{1}$ Queen's University Belfast, School of Psychology, Belfast BT7 1NN, United Kingdom, and 2Trinity College Dublin, School of Psychology and Trinity College \\ Institute of Neuroscience, Dublin 2, Ireland
}

\begin{abstract}
The effect of vision on the excitability of corticospinal projections to the flexor carpi radialis (FCR) and extensor carpi radialis (ECR) muscles of right human forearm was investigated before and during discrete movement of the opposite limb. An external force opposed the initial phase of the movement (wrist flexion) and assisted the reverse phase, so that recruitment of the wrist extensors was minimized. Three conditions were used as follows: viewing the inactive right limb (Vision), viewing the mirror image of the moving left limb (Mirror), and with vision of the right limb occluded (No Vision). Transcranial magnetic stimulation was delivered to the left motor cortex: before, at the onset of, or during the left limb movement to obtain motor evoked potentials (MEPs) in the muscles of the right forearm. At and following movement onset, MEPs obtained in the right FCR were smaller in the Vision condition than in the Mirror and No Vision conditions. A distinct pattern of variation was obtained for the ECR. In all conditions, MEPs in this muscle were elevated upon or following movement of the opposite limb. An additional analysis of ipsilateral silent periods indicated that interhemispheric inhibition plays a role in mediating these effects. Activity-dependent changes in corticospinal output to a resting limb during discrete actions of the opposite limb are thus directly contingent upon where one looks. Furthermore, the extent to which vision exerts an influence upon projections to specific muscles varies in accordance with the functional contribution of their homologs to the intended action.
\end{abstract}

\section{Introduction}

In the course of our daily lives, we routinely perform actions in which distinct patterns of movement are required of the two hands. The apparent ease with which we unscrew the lid from a jar, or thread a needle, belies the fact that there is a tendency for movements of the upper limbs to be drawn toward one another (Swinnen, 2002). For isolated independent actions to be performed, such tendencies must be mitigated.

The origin of the disposition for simultaneity of action is thought to be facilitatory interhemispheric interactions between cortical motor areas (Carson et al., 2004). The presence of countermanding inhibition has been inferred from reports that a conditioning transcranial magnetic stimulation (TMS) pulse directed to one primary motor cortex (M1) shortly before a second (test) stimulus is applied to the other, diminishes the corticospinal volley generated by the test stimulus (Ferbert et al., 1992). The balance of evidence indicates that callosal neurons are facilitatory to their immediate targets (Houzel and Milleret, 1999). Thus, the extent to which the output of one cortical motor network invokes crossed inhibition is contingent on

\footnotetext{
Received Aug. 30, 2011; revised Nov. 15, 2011; accepted Nov. 21, 2011.

Author contributions: R.G.C. designed research; K.L.R. performed research; R.G.C. contributed unpublished reagents/analytic tools; R.G.C. and K.L.R. analyzed data; R.G.C. wrote the paper.

We thank Billy Neil, Fred Maddalena, Chris White, and Paul Coulter for technical assistance.

Correspondence should be addressed to Richard G. Carson, School of Psychology and Trinity College Institute of Neuroscience, Trinity College Dublin, Dublin 2, Ireland. E-mail: richard.carson@tcd.ie.

DOI:10.1523/JNEUROSCI.4435-11.2012

Copyright $\odot 2012$ the authors $\quad 0270-6474 / 12 / 320646-07 \$ 15.00 / 0$
}

neural interactions that converge upon circuits local to the opposite hemisphere (Daskalakis et al., 2002; Carson, 2005). The ensuing degree of corticospinal suppression is therefore likely to be influenced by inputs from numerous brain centers and exhibit task dependency.

Preceding the onset of a discrete movement, there is a transient decrease in the excitability of corticospinal projections to the muscles of the opposite limb (Duque et al., 2005). This effect exhibits a high degree of kinematic specificity: the descending projections to a given muscle are most deeply inhibited in (postural) contexts in which the recruitment of that muscle would produce motion mirroring that of the active limb. In rhythmic tasks, the inhibitory/facilitatory balance of (M1) cortical output directed to the opposite limb is modified by augmented (Carson et al., 2005) and mirrored (Garry et al., 2005) visual feedback of the moving limb.

We hypothesized that, during unilateral movement, vision of the opposite limb would mitigate crossed facilitation. We also predicted that the extent of its influence upon the projections to specific muscles would vary in accordance with the functional contribution of their homologs to the intended action. To examine these hypotheses, we used a task in which an initial phase (wrist flexion) was opposed by an external force and a reverse phase assisted by the force, so that recruitment of the wrist extensors was minimized. TMS was used to assess the excitability of corticomotor projections to the opposite limb in three conditions: viewing the inactive limb, with vision of the inactive limb occluded, and viewing the mirror image of the moving limb su- 


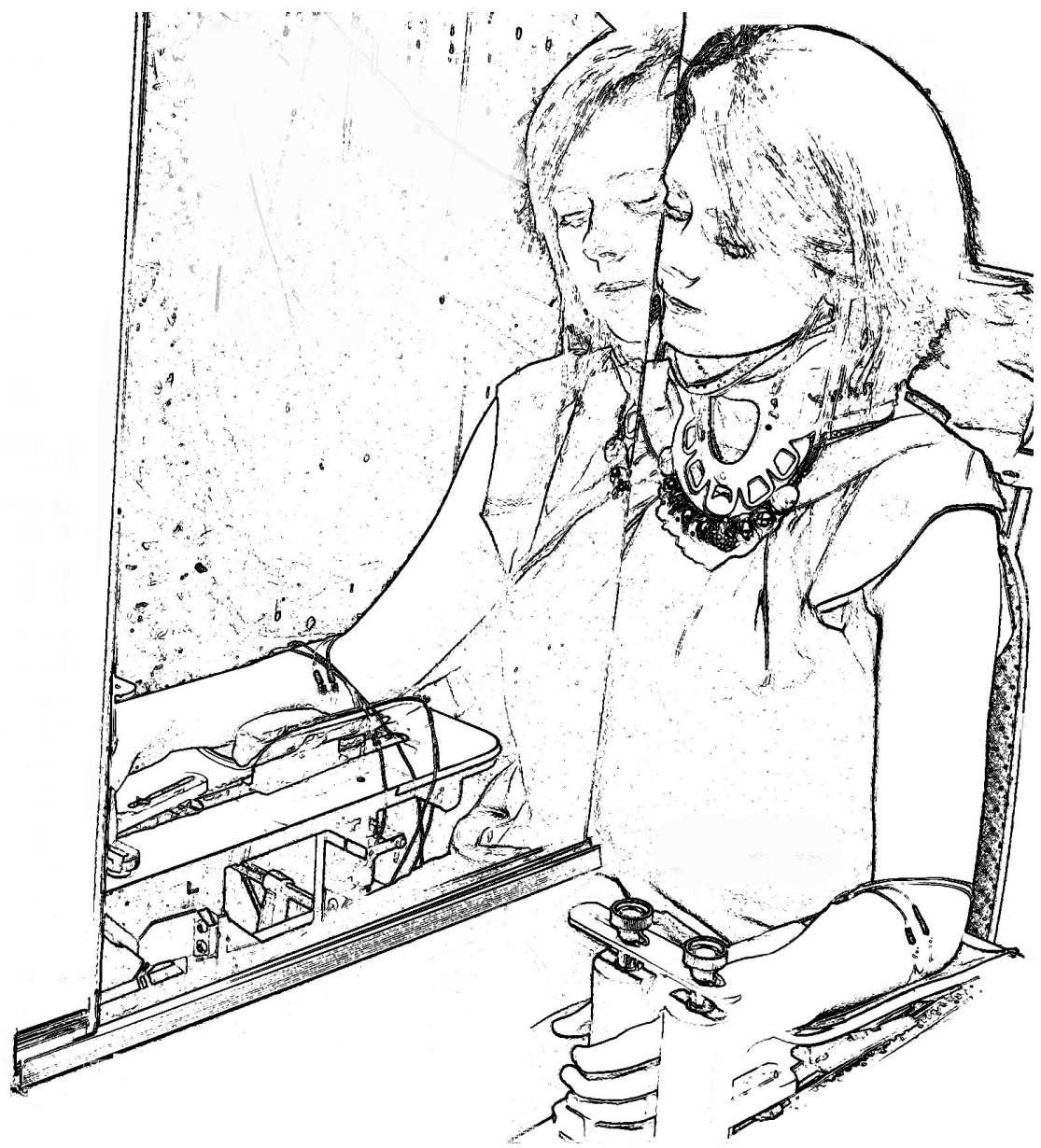

Figure 1. Experimental setup. The participant is shown in the Mirror condition, positioned such that the reflection of the left limb is superimposed precisely upon the directly sighted position of the right limb. In the No Vision condition, a drape was placed in front of the mirror, eliminating both the reflected image of the left limb and direct vision of the right limb. A white cross on the drape served as a point of fixation. In the Vision condition, the mirror was withdrawn, affording direct vision of the right arm.

relative to a change in the status of the switch by a microcomputer running custom software routines.

A mirror (depth by height, $50 \times 90 \mathrm{~cm}$ ) was aligned with the participant's sagittal plane. As the mirror was incompletely silvered, it could first be positioned to ensure that the reflection of the left limb was superimposed precisely upon the directly sighted position of the right limb. When a drape was placed behind the mirror, the partial transparency was eliminated (Mirror condition). The mirror could also be withdrawn, affording direct vision of the right arm (Vision condition). Placing the drape in front of the mirror eliminated both the reflected image of the left limb and direct vision of the right limb (No Vision condition). A white cross on the drape served as a point of fixation during trials conducted in the latter condition.

The electromyographic (EMG) activity of flexor carpi radialis (FCR) and extensor carpi radialis longus (ECR) was recorded from both arms using bipolar surface electrodes. EMG signals were amplified and bandpass $(30 \mathrm{~Hz}$ to $1 \mathrm{kHz}$ ) filtered. These signals and the transducer-derived voltage corresponding to displacement of the left wrist were digitized at $5000 \mathrm{~Hz}$.

Magnetic stimuli were delivered to the left primary motor cortex (M1) by a Magstim 200 stimulator using a (55 mm mid-diameter) figure of eight coil, located at the optimal position ("hot spot") to obtain a motor evoked potential (MEP) in the FCR muscle of the contralateral (right) arm. The coil was placed so that the axis of intersection between the two loops was oriented at $\sim 45^{\circ}$ to the sagittal plane, to induce posterior-to-anterior current flow across the motor strip. Once the hot spot was established, the lowest stimulation intensity at which MEPs with peak-to-peak amplitude of $\sim 50 \mu \mathrm{V}$ were evoked in at least 5 of 10 consecutive trials was

perimposed over the inactive limb. We found that direct vision of the inactive limb markedly reduced crossed facilitation. Critically, this effect was specific to the homolog of the primary agonist (the wrist flexor). No equivalent diminution was present for the homolog of the wrist extensor, which was not strongly engaged in the task.

\section{Materials and Methods}

Participants. In Experiment 1, 12 healthy volunteers (age, 21.5 \pm 3.4 SD; eight females) participated in three testing sessions. Thirteen participants (age, 25.0 \pm 4.0 SD; seven females) were included in the second experiment. Five individuals took part in both experiments. The participants (all right-handed) gave informed consent to the procedures, approved by the Queen's University Belfast Ethics Committee and conducted in accordance with the Declaration of Helsinki.

Apparatus and procedures. The participants were seated with forearms supported and stabilized in a neutral position with the elbows semiflexed $\left(100-120^{\circ}\right)$. The angle between the upper arm and the torso was $15-20^{\circ}$. An orthopaedic neck brace stabilized the head at $\approx 15^{\circ}$ relative to the saggital plane. A constant position of the head was thereby maintained in all experimental conditions (Fig. 1).

The hands were secured at midpalm in manipulanda (instrumented to transduce angular displacement) mounted coaxially with the (flexion-extension) axes of rotation of the wrists. A contact switch was activated upon flexion of the left wrist (from a neutral position), which was opposed by a stiffness load $(\approx 0.67 \mathrm{Nm} / \theta-\mathrm{rad})$. The delivery of TMS was timed precisely taken as resting motor threshold (RMT). The level of stimulation used subsequently was $120 \%$ of the FCR RMT. At this intensity a potential was also evoked in the ECR. To obtain an ipsilateral silent period (iSP) (Experiment 2), TMS was delivered in some trials at $80 \%$ of the maximum output of the stimulator (Jung and Ziemann, 2006). At the beginning and at the end of each testing session, three sets of 10 control MEPs were obtained while the participant sat quietly.

Experimental paradigm. The participant was required to initiate a discrete flexion movement of the left wrist upon presentation of a tone (500 $\mathrm{Hz}$ sin wave), and to return to the starting position (i.e., wrist neutral) before the onset of a second tone that followed 1000 ms later. In Experiment 1,12 such movements were performed during the course of a trial (intervals $\approx 4000 \mathrm{~ms}$ ). In eight of these, TMS at $120 \%$ FCR RMT was delivered to the left motor cortex: $450 \mathrm{~ms}$ before the first tone (" $450 \mathrm{~ms}$ premovement"), or at movement onset, or $100 \mathrm{~ms}$ after onset, or $200 \mathrm{~ms}$ after onset. In Experiment 2, 15 movements were performed in each trial. During six of these, TMS at $120 \%$ FCR RMT was delivered to the left motor cortex: $200 \mathrm{~ms}$ before the first tone ("200 ms premovement"), or at movement onset, or $100 \mathrm{~ms}$ after onset. During four others, TMS at $80 \%$ of maximum stimulator output was delivered to the left motor cortex either at movement onset or $100 \mathrm{~ms}$ after onset. The order in which these alternatives were presented (two instances of each) was randomized. There were 10 trials in total, separated by 1 min intervals. In each session, five practice trials were first undertaken to familiarize the participant with the procedure. 
In the Mirror condition, the participants were asked to complete the task while looking at the reflection of their moving left limb. In the Vision condition, the participants were instructed to look at their inactive right limb while executing the movements with their left limb. In the No Vision condition, the participants were required to look at the white fixation cross while executing unseen the movements of their left limb. Testing sessions, which each comprised a single condition, were separated by at least 1 week. The order of conditions was counterbalanced across participants.

Data analyses. Due to a technical problem undetected at the time of collection, data from one participant in Experiment 1 could not be used. For the others, the peak-to-peak amplitude of the MEPs recorded in the right FCR and ECR, and the root-mean-squared (RMS) amplitude of the EMG signal recorded $100 \mathrm{~ms}$ before the delivery of each stimulus, were calculated both for trials in which the opposite limb moved, and for the control trials in which the participant sat quietly. If the RMS EMG in either muscle exceeded $5 \mu \mathrm{V}$, the corresponding MEPs for both muscles were excluded from further consideration. The data from two participants in Experiment 1 were removed from the analysis, as in each case for a single muscle in a single session this threshold was exceeded marginally. In the movement trials, the mean signal amplitude of the retained data ( 9 of the original 12 participants) was 2.6 and $3.0 \mu \mathrm{V}$ for the FCR and ECR, respectively. For Experiment 2 (13 participants), the corresponding values were 2.0 and $2.0 \mu \mathrm{V}$ for the FCR and ECR, respectively. In no instance was there a statistically reliable difference between the experimental conditions.

MEP amplitudes were normalized with respect to the magnitude of the responses obtained at the beginning and at the end of each testing session. To reduce skewness, these ratios were subjected to an arcsine transformation (Myers, 1979). To align the inferential tests with the experimental hypotheses, and to maximize statistical power, planned comparisons of means were calculated using a repeated-measures ANOVA design (Keppel, 1991). In this regard, and in line with the a priori hypotheses, MEP responses in the mirror condition were compared with the Vision and No Vision conditions, respectively. The omnibus $F$ tests were not evaluated.

The modified Bonferroni adjustment for multiple comparisons (Keppel, 1991) was applied. The aim of this procedure is to control for the potential elevation of familywise error associated with multiple comparisons. The implicitly assumed familywise error is obtained as the product of the degrees of freedom (df) and the $\alpha$ level (conventionally 0.05). In the event that the theoretically motivated set of planned comparisons exceeds the df, it is necessary to adjust the effective level of $\alpha$ to preserve the familywise error rate. In Experiment 1, this resulted in a criterion $\alpha$ probability of 0.037 , and in Experiment 2, a value of 0.033 .

An effect size $(f)$ was calculated for each planned comparison following Cohen (1988). The effect size index for ANOVA $(f)$ is a dimensionless index, which describes the degree of departure from no effect, in other words, the degree to which the phenomenon is manifested. A small effect size is considered by convention to be indicated by an $f$ of 0.1 , a medium effect size by an $f$ of 0.25 , and a large effect size by an $f$ of 0.4 . To establish whether the responses in each condition differed from controls, 99\% confidence intervals (Loftus and Masson, 1994) were obtained. A difference was deemed to be present when the test value deviated from unity (i.e., the magnitude of the control response) by more than the confidence interval.

\section{Results}

\section{Movements of the left arm}

These analyses were performed using movements (25\%) not associated with TMS stimuli. Following digital filtering (secondorder, dual-pass Butterworth, low pass, $6 \mathrm{~Hz}$ ), the duration and excursion of each flexion movement (Fig. 2) was determined using standard procedures (Teasdale et al., 1993). There were no reliable differences in mean movement excursion across the experimental conditions in either Experiment 1 (Mirror, 55.7 $7^{\circ}$ Vision, $55.3^{\circ}$; No Vision, $\left.53.8^{\circ} ; F_{(2,16)}<1, p>0.20\right)$ or Experiment 2 (Mirror, $60.3^{\circ}$; Vision, $58.7^{\circ} ; F_{(1,12)}<1, p>0.20$ ). There were no systematic variations in movement duration in Experiment 1

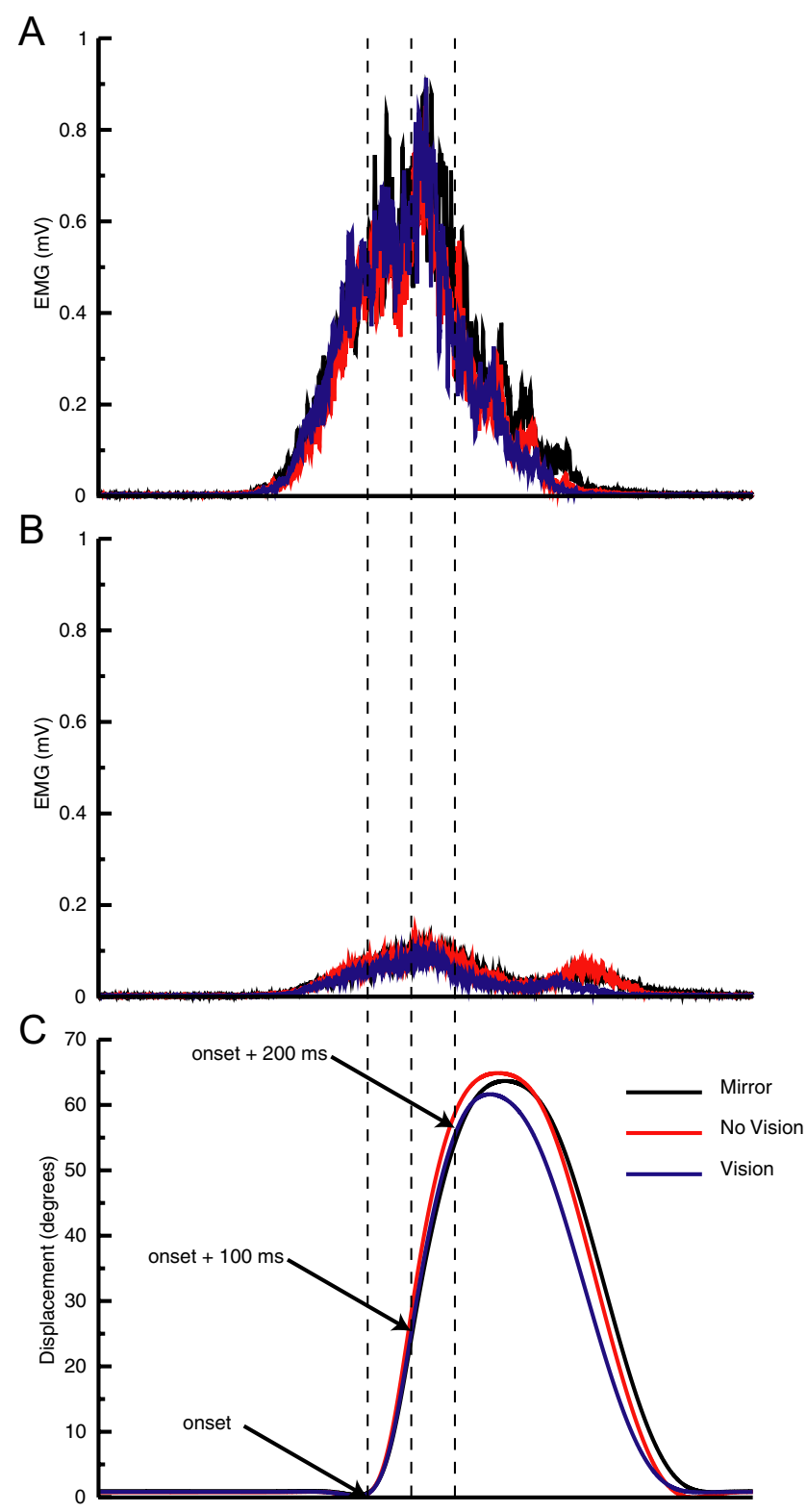

Figure 2. Averaged kinematic and rectified EMG profiles ( $n=40$ movements) obtained for one participant during flexion movements of the left wrist in the Mirror (black trace), Vision (red trace), and No Vision (blue trace) conditions in Experiment 1. Epochs of 1.5 s duration are shown ( 0.6 s before; 0.9 s following movement onset). $\boldsymbol{A}$, RMS EMG recorded from left FCR. $\boldsymbol{B}$, RMSEMG recorded from left ECR. $C$, Excursion of the left wrist. The dashed vertical lines represent times at which cortical stimulation was delivered to the left motor cortex during the remaining $(n=80)$ movements in each condition. The "at rest" time of stimulation is not shown.

(Mirror, $686 \mathrm{~ms}$; Vision, $662 \mathrm{~ms}$; No Vision, $662 \mathrm{~ms} ; F_{(2,16)}=$ $1.62, p>0.20)$. Movements performed in the Vision $(720 \mathrm{~ms})$ condition in Experiment 2 were of somewhat longer duration than those generated in the Mirror $(662 \mathrm{~ms})$ condition $\left(F_{(1,12)}=\right.$ 7.41; $p<0.05)$.

The onsets of EMG burst activity (Fig. 2) in the muscles of the left arm were obtained using methods described previously (Carson et al., 2002). For each muscle, the RMS amplitude of the EMG signal in the period from the onset of its activity to $200 \mathrm{~ms}$ following the start of the movement was calculated. The level of EMG activity recorded in the left FCR was substantially greater than that present in left ECR (Fig. 2). This is in accordance with its role in generating force in opposition to the external load, 

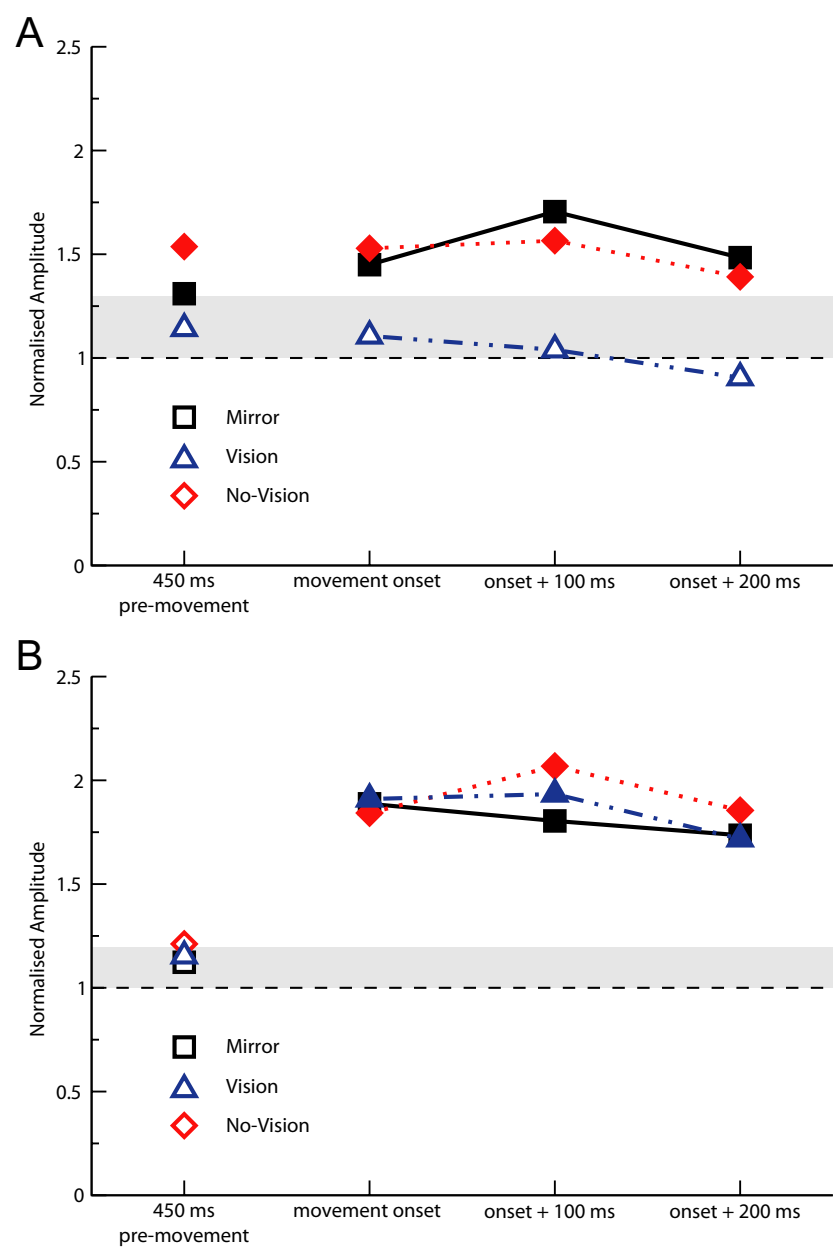

Figure 3. Experiment 1. Amplitudes of compound muscle action potentials evoked in the quiescent right $\mathrm{FCR}(\boldsymbol{A})$ and right $\mathrm{ECR}(\boldsymbol{B})$ by left M1 stimulation: 450 ms before, at the onset of, and following (100 and $200 \mathrm{~ms}$ ) the onset of flexion movements of the left wrist. Values are normalized with respect to controls (left limb static) obtained before and following the movement trials. Mean responses ( $n=9$ participants) in the Mirror condition are shown as squares (solid black lines), in the No Vision condition as diamonds (dotted red lines), and in the Vision condition as triangles (dash-dotted blue lines). The $99 \%$ confidence intervals are represented as the gray shaded area. Instances in which values deviated from the control response by more than the magnitude of the confidence intervals are shown as filled symbols.

which opposed flexion of the wrist. Inferential analyses of these data failed to reveal the presence of systematic variations across conditions for either the FCR (Experiment 1: Mirror, $464 \mu \mathrm{V}$; Vision, $514 \mu \mathrm{V}$; No Vision, $441 \mu \mathrm{V} ; F_{(2,16)}<1, p>0.20$; Experiment 2: Mirror, $254 \mu \mathrm{V}$; Vision, $\left.258 \mu \mathrm{V} ; F_{(1,12)}<1, p>0.20\right)$ or ECR (Experiment 1: Mirror, $67 \mu \mathrm{V}$; Vision, $72 \mu \mathrm{V}$; No Vision, 72 $\mu \mathrm{V} ; F_{(2,16)}<1, p>0.20$; Experiment 2: Mirror, $32 \mu \mathrm{V}$; Vision, 31 $\left.\mu \mathrm{V} ; F_{(1,12)}<1, p>0.20\right)$ muscles.

\section{Potentials evoked in the right arm}

In the absence of systematic variations in the kinematic or electromyographic characteristics of the movements generated by the left arm, differences between experimental conditions in relation to the excitability of corticospinal projections to the muscles of the right arm can be attributed to the manipulation of vision.

\section{Experiment 1}

As Figure $3 A$ illustrates, during movement of the opposite limb, when the participants looked directly at the static right arm (Vi- sion), MEPs elicited from right FCR were markedly lower than in the condition (Mirror) in which they viewed the mirror image of the moving (left) limb. This effect was expressed reliably $(p<$ 0.037 ;f $=1,48) 100 \mathrm{~ms}$ after onset $(F=15.02 ; f=0.91)$, and at $200 \mathrm{~ms}$ after onset $(F=14.36 ; f=0.89)$, but not before movement $(F=1.12 ; p>0.20)$, and to a lesser degree at movement onset $(F=3.71 ; f=0.45)$. For all time points, the MEP amplitudes obtained in the Mirror condition were not differentiated from those recorded in the No Vision condition (before movement onset, $F=2.81, p=0.1$; otherwise, $F<1, p>0.20)$. When the right arm was viewed directly (Vision), the MEP amplitudes were not differentiated from the control responses obtained at the beginning and at the end of each testing session. In contrast, those obtained in the Mirror and No Vision conditions were in all cases elevated above control values.

A quite distinct pattern of variation was obtained for the ECR (Fig. 3B). Upon and following movement of the opposite limb, in all experimental conditions, MEPs were elevated reliably relative to control responses. There were, however, no reliable differences $\left(F_{(1,48)}=0.01-0.78 ; p>0.20\right)$ in amplitude between the Vision and Mirror conditions. Similarly, the Mirror and No Vision conditions were largely undifferentiated $\left(F_{(1,48)}<1 ; p>0.20\right)$, other than at $100 \mathrm{~ms}$ following the onset of movement $\left(F_{(1,48)}=4.6\right.$; $p=0.037 ; f=0.51$ ), for which values obtained in the No Vision condition were slightly larger than those recorded in the Mirror condition.

Before the onset of movement of the opposite limb, the amplitudes of MEPs recorded from ECR in the Vision and No Vision conditions were not differentiated reliably from those in the Mirror condition $\left(F_{(1,48)}=0.14\right.$ and 1.0 , respectively; $\left.p>0.20\right)$. At this time point the values obtained in the No Vision condition (1.21) were elevated somewhat relative to controls.

\section{Experiment 2}

Two of the previous manipulations of visual feedback (Vision and Mirror) were included. In respect of these conditions, the outcomes of the second experiment were in accordance with those obtained previously. When the participants looked directly at the static right arm (Vision), MEPs elicited from right FCR (Fig. 4A) were markedly lower than in the condition (Mirror) in which they viewed the mirror image of the moving (left) limb $(p<0.033 ; \mathrm{df}=1,24)$, both at movement onset $(F=23.76 ; f=$ $0.96)$ and $100 \mathrm{~ms}$ after onset $(F=8.83 ; f=0.58)$. No such differences were apparent before movement $(F=1.07 ; p>0.20)$.

As in Experiment 1, the amplitudes of MEPs evoked in ECR in the Mirror and Vision conditions were differentiated to a much lesser degree. There were no reliable $(\mathrm{df}=1,24)$ differences in MEP amplitude between conditions either before movement $(F=3.40 ; p=0.08)$ or $100 \mathrm{~ms}$ after onset $(F=1.60 ; p>0.20)$. It was, however, the case that at the time of movement onset, the values obtained in the Mirror condition were larger than those present in the Vision condition $(F=11.42 ; p<0.033 ; f=0.66)$. In the previous experiment, MEPs elicited $450 \mathrm{~ms}$ before the imperative stimulus (the first tone) could not be distinguished from controls. Whereas when evoked $200 \mathrm{~ms}$ in advance of the tone in Experiment 2, there was an elevation relative to controls of the values obtained for both FCR and ECR.

iSP

An automated method (Chen et al., 2003) was used to establish the time of onset, time of offset, and the duration of the silent period for ensemble averages ( $n=20$ responses) of the rectified EMG, constructed separately for each participant in each combi- 

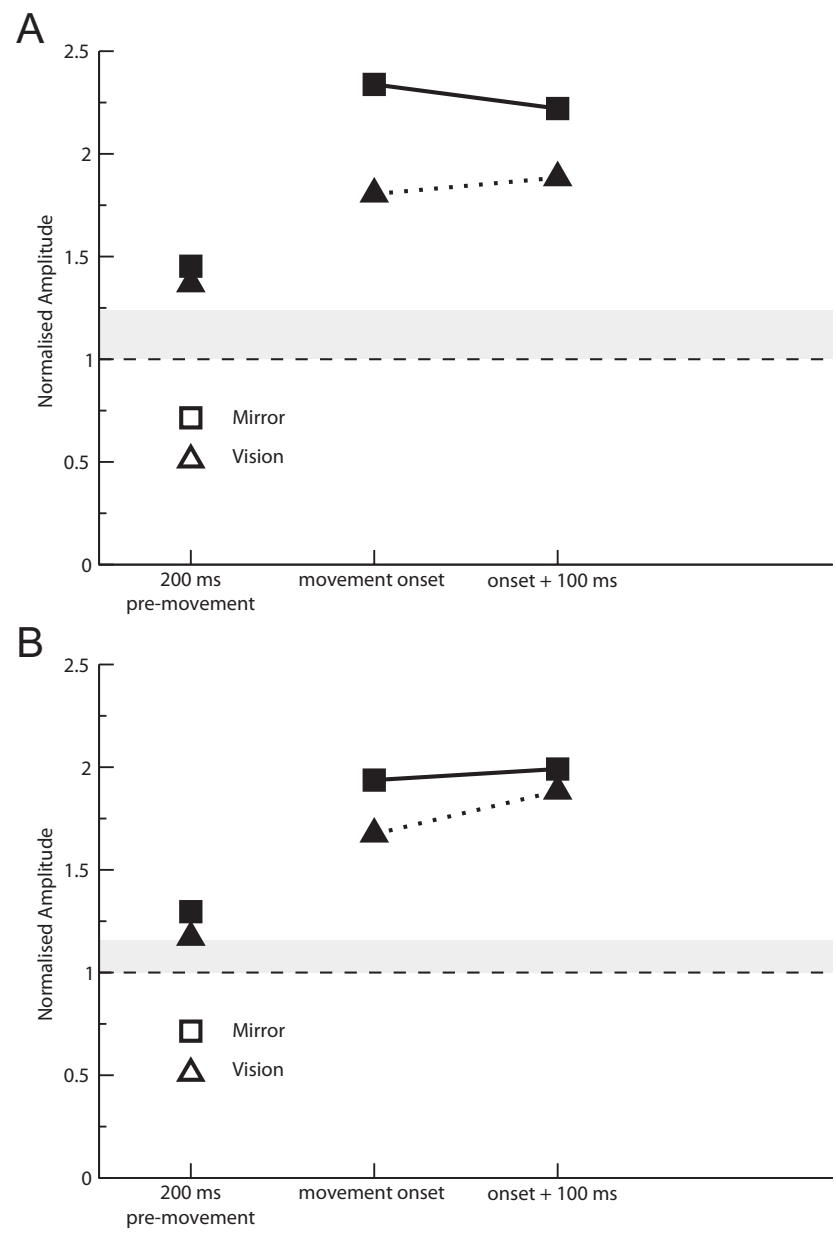

Figure 4. Experiment 2. Amplitudes of compound muscle action potentials evoked in the quiescent right $\mathrm{FCR}(\boldsymbol{A})$ and right $\mathrm{ECR}(\boldsymbol{B})$ by left M1 stimulation: $200 \mathrm{~ms}$ before, at the onset of, and following $(100 \mathrm{~ms})$ the onset of flexion movements of the left wrist. Values are normalized with respect to controls (left limb static) obtained before and following the movement trials. Mean responses ( $n=13$ participants) in the Mirror condition are shown as squares (solid lines), and in the Vision condition as triangles (dotted lines). The $99 \%$ confidence intervals are represented as the gray shaded area. Instances in which values deviated from the control response by more than the magnitude of the confidence intervals are shown as filled symbols.

nation of condition (Mirror, Vision) and timing of TMS (movement onset, movement onset plus $100 \mathrm{~ms}$ ). In a further step, we calculated the level of EMG (RMS) activity during the silent periods thus defined, and expressed these values relative to the level of EMG obtained in the same epochs during trials in which no TMS was delivered ( $n=50$ responses). Although the level of EMG activity recorded from the left ECR was substantially lower than that present for the FCR, silent periods were evident in both cases.

There were no reliable differences between the two conditions in terms of the time of onset, time of offset, or duration of the silent periods for either FCR or ECR. The degree to which FCR EMG activity was suppressed during the silent period (Fig. 5B) was, however, greater in the Vision condition (53.3\%) than in the Mirror condition (45.3\%), when stimulation was delivered at the time of movement initiation $\left(F_{(1,12)}=8.14 ; p<0.02 ; f=0.56\right)$. There tended also to be less suppression of ECR EMG in the Vision condition than in the Mirror condition, when the iSP was generated $100 \mathrm{~ms}$ after the onset of movement $\left[F_{(1,12)}=5.08 ; p=\right.$ 0.044 ( $>$ adjusted $\alpha=0.02$ ); $f=0.44$ ].
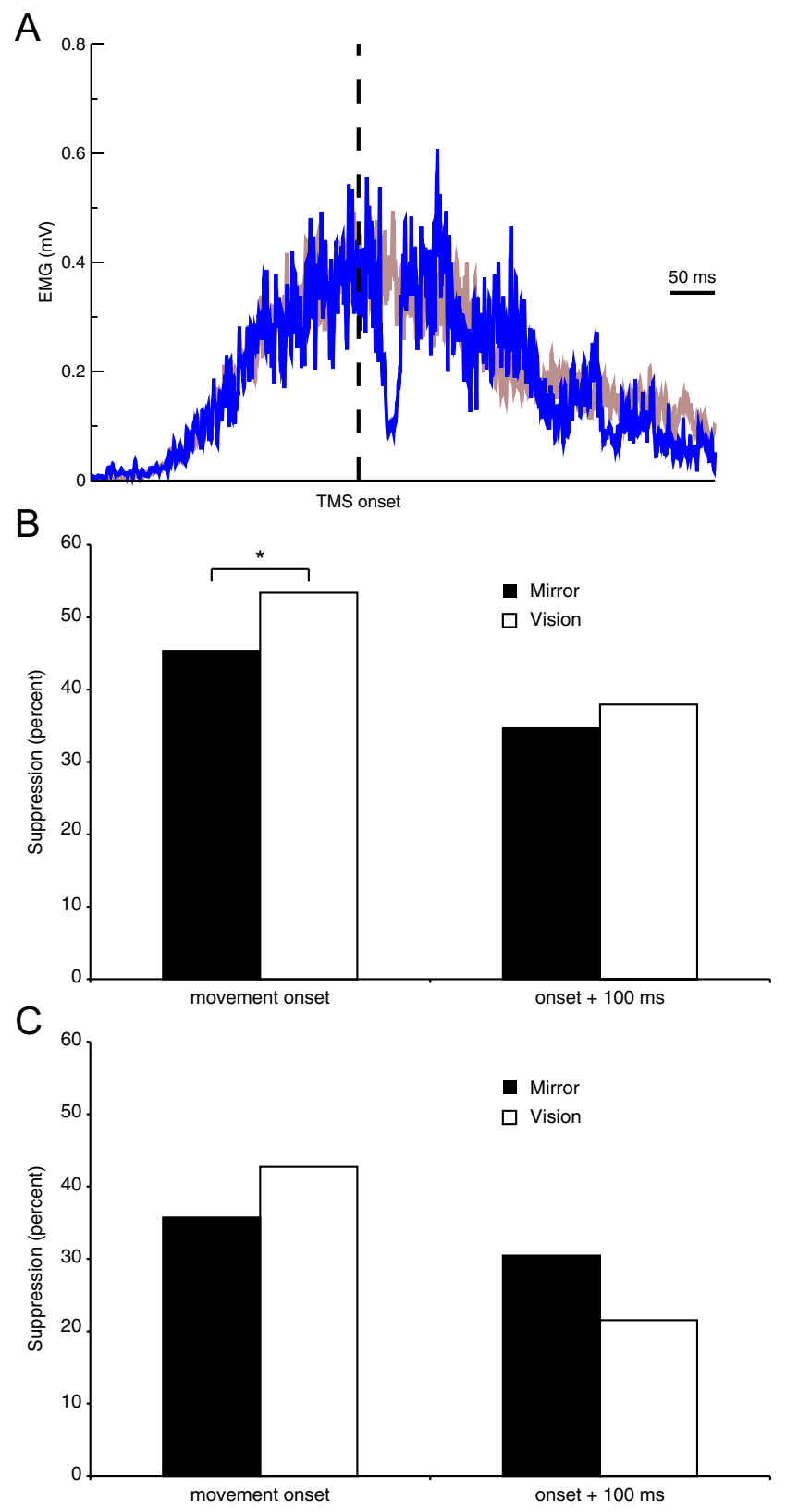

Figure 5. Experiment 2. In $\boldsymbol{A}$, the averaged rectified FCR EMG profiles obtained for one participant during flexion movements of the left wrist in the Vision condition are shown for trials in which no TMS was delivered (brown trace: $n=50$ movements), and for trials in which TMS at $80 \%$ of the stimulator output was delivered at the onset of movement (blue trace: $n=20$ movements). Mean ( $n=13$ participants) suppression of RMS EMG during the iSP in left FCR ( $\boldsymbol{B})$ and left ECR ( $C$ ) computed as a percentage of the level of activity present during trials in which no TMS was delivered. Larger values indicate greater suppression. The iSP was generated either at movement onset or $100 \mathrm{~ms}$ following movement onset (open bars, Vision; filled bars, Mirror). The asterisk $\left(^{*}\right)$ indicates the presence of a statistically reliable $(p<0.02)$ contrast between the experimental conditions.

\section{Discussion}

Mirror movements arise from involuntary contractions during intended unilateral engagement of the opposite limb. The pathological conditions that give rise to obligate mirror movements are heterogenous, both with respect to etiology and to the neural pathways that mediate their expression. They are, however, observed in normally developing children up to the age of 8 or 9 years, their prevalence and magnitude decreasing thereafter (Abercrombie et al., 1964; Connolly and Stratton, 1968; Mayston et 
al., 1999). While in the mature motor system the contraction of muscles on one side of the body can occasion increases in the excitability of the (opposite) homologous motor pathway, and promote simultaneity of action, the overt expression of unintended mirror movements is abnormal, suggesting that in most cases compensating inhibitory processes are sufficient to mitigate the effects of crossed facilitation. The objective of the present study was to determine the role of vision in these processes, and to examine the hypothesis that visual mediation of corticospinal suppression is most prominent for homologs of the muscles that are functionally engaged in the intended action.

We used a task in which the initial phase (wrist flexion) of a discrete voluntary movement was opposed by an external force. A significant level of engagement of the wrist flexor muscles was thus required. Conversely, as the phase in which the limb returned from the flexed to a neutral position was assisted by the force, recruitment of the wrist extensor muscles was minimized. When vision of the inactive limb was occluded (Experiment 1), or the mirror image of the (opposite) moving limb was viewed (Experiments 1 and 2), the flexion movements gave rise to increases in the excitability of descending projections to the homologous (FCR) muscle. The key novel finding was that such crossed facilitation was markedly diminished in the Vision condition in which the inactive limb was viewed directly.

The effective suppression of excitatory callosal inputs during movement of the ipsilateral limb is thought to be due to the action of local inhibitory interneurons. It has been proposed that these are subthreshold during unimanual movements, and recruited during bimanual movements in response to control signals from other brain areas (Rokni et al., 2003). The results of our first experiment promoted the conclusion that, when vision is directed to the inactive limb, inhibitory circuits are engaged during unimanual movements. To examine this issue further, in Experiment 2 we used a method of assessing interhemispheric inhibition based on quantification of the iSP. This is typically obtained when TMS is delivered at high intensity to the M1 ipsilateral to contracting muscles (Wassermann et al., 1991). As the iSP is thought to be mediated, at least in part, by the fibers of the corpus callosum (Meyer et al., 1995, 1998), its extent is influenced by processes of transcallosal inhibition. It provides information that is complementary to the measures of interhemispheric inhibition that have been derived using paired-pulse TMS techniques (Chen et al., 2003), and is particularly well suited to the investigation of interhemispheric control in the context of voluntary cortical motor output (Giovannelli et al., 2009).

The results obtained from the component of Experiment 2 in which crossed facilitation was assessed in the manner of the previous experiment, confirmed that the magnitude of this effect was markedly lower when the inactive limb was viewed than when the mirror image of the moving limb was seen. As indicated by the relative size of the Cohen's effect size indices, the difference between the two conditions was most pronounced at the initiation of movement. It was therefore noteworthy that, when highintensity TMS was applied to ipsilateral M1 at this time, the degree to which FCR EMG activity was suppressed (i.e., the magnitude of the iSP) was greater in the Vision condition than in the Mirror condition. While it is not possible on the basis of the iSP methodology alone to delineate further the processes underlying the visual feedback-related variations in corticospinal excitability, we believe that the results of the second experiment provide a clear indication that mechanisms involved in the mediation of interhemispheric inhibition are implicated.
Although it appears that cortical suppression is most pronounced for prime movers, the nature of the present task required that this role be accorded always to the wrist flexors. There is a need to establish whether this effect of vision extends to tasks in which other muscle groups assume a more prominent role, and to those in which there is sensitivity to the direction of muscle action (Post et al., 2009). That which also remains to be elucidated is the means by which input from those parts of the brain network that process visual feedback, provides the requisite control signals to regulate motor output.

The dorsal premotor cortex (PMd) plays a significant role in visuomotor integration during upper limb movements (Wise et al., 1997). In macaque, neurons in premotor cortex also modulate their firing frequency in the preparatory phase of arm movements guided by vision. Some of these neurons receive monosynaptic projections from the inferior parietal lobule, which forms part of the parietal association cortex, and receives input from numerous visual areas (Godschalk et al., 1985). In relation to motor output, it is thought that premotor cortex assumes a focusing role by modulating the activity of interneurons in M1 (Münchau et al., 2002). Recent studies using TMS (and combined fMRI) in humans suggest a functional influence of (left) PMd upon the opposite PMd (and M1) that is strongly state dependent: varying in accordance with the nature of the task being performed by the ipsilateral limb (Bestmann et al., 2008). During movement preparation, there is a corresponding influence of (right) PMd on opposite M1, which precedes interhemispheric interactions between the homologous primary motor areas (Liuzzi et al., 2010). It is possible, therefore, that the state (i.e., vision)-dependent modulation of crossed facilitation observed in the present study was mediated via differential PMd control of interneuronal (i.e., inhibitory) circuitry within left and right $\mathrm{M} 1$.

The close equivalence of the pattern of outcomes obtained in the No Vision and Mirror conditions in Experiment 1 suggests that the distinct effects observed in the Vision condition were not simply attributable to differences in the locus of spatial attention. In particular, the mirror was positioned such that the reflection of the left limb was superimposed precisely upon the directly sighted position of the right limb, thus ensuring that the locus of attention was equivalent in the Vision and Mirror conditions.

It is striking that, in all visual feedback conditions, there was a marked increase in the excitability of the corticospinal projections to the wrist extensor during the flexion movement of the opposite limb. At first glance, this may seem counterintuitive, as the ECR muscle of the moving limb was not engaged to a functionally significant degree. Sohn et al. (2003) reported a distal to proximal gradient of crossed inhibition, whereby the suppression of corticospinal excitability in projections to muscles (other than homologs of the prime mover) opposite to the moving limb, was greater for muscles of the fingers than for a muscle of the upper arm. In Experiment 2, the interval between the premovement stimulus and the imperative signal was 200 ms, whereas in Experiment 1 it was $450 \mathrm{~ms}$. It was thus interesting to note that in the second experiment, for both FCR and ECR, MEPs were elevated above the confidence intervals defined by the control values obtained before and following the movement trials. As this was not the case in the first experiment, these outcomes suggest that there was an increase of corticospinal excitability in anticipation of the forthcoming movement of the opposite limb. Consideration might therefore be given to the potential functional role of a preparatory set that encompasses an increase in the excitability of descending projections to agonist-antagonist pairs (i.e., FCR and 
ECR). Sherrington (1909) proposed that there are forms of cerebral action, otherwise latent, that through "double reciprocal innervation" can give rise to agonist-antagonist co-contraction, the balance of these being such that joint stiffness and stability is increased, and the maintenance and monitoring of posture promoted (Smith, 1981). The possibility exists that the presently observed simultaneous increase in the excitability of corticospinal projections to ECR and FCR before flexion movements of the opposite wrist reflects a preparatory set tailored preemptively for the prevention of mirror movement.

\section{References}

Abercrombie ML, Lindon RL, Tyson MC (1964) Associated movements in normal and physically handicapped children. Dev Med Child Neurol 6:573-580.

Bestmann S, Swayne O, Blankenburg F, Ruff CC, Haggard P, Weiskopf N, Josephs O, Driver J, Rothwell JC, Ward NS (2008) Dorsal premotor cortex exerts state-dependent causal influences on activity in contralateral primary motor and dorsal premotor cortex. Cereb Cortex 18:1281-1291.

Carson RG (2005) Neural pathways mediating bilateral interactions between the upper limbs. Brain Res Brain Res Rev 49:641-662.

Carson RG, Smethurst CJ, Forner M, Meichenbaum DP, Mackey DC (2002) Role of peripheral afference during acquisition of a complex coordination task. Exp Brain Res 144:496-505.

Carson RG, Riek S, Mackey DC, Meichenbaum DP, Willms K, Forner M, Byblow WD (2004) Excitability changes in human forearm corticospinal projections and spinal reflex pathways during rhythmic voluntary movement of the opposite limb. J Physiol 560:929-940.

Carson RG, Welsh TN, Pamblanco-Valero MA (2005) Visual feedback alters the variations in corticospinal excitability that arise from rhythmic movements of the opposite limb. Exp Brain Res 161:325-334.

Chen R, Yung D, Li JY (2003) Organization of ipsilateral excitatory and inhibitory pathways in the human motor cortex. J Neurophysiol 89: $1256-1264$

Cohen J (1988) Statistical power analysis for the behavioral sciences. Hillsdale, NJ: Lawrence Erlbaum.

Connolly K, Stratton P (1968) Developmental changes in associated movements. Dev Med Child Neurol 10:49-56.

Daskalakis ZJ, Christensen BK, Fitzgerald PB, Roshan L, Chen R (2002) The mechanisms of interhemispheric inhibition in the human motor cortex. J Physiol 543:317-326.

Duque J, Mazzocchio R, Dambrosia J, Murase N, Olivier E, Cohen LG (2005) Kinematically specific interhemispheric inhibition operating in the process of generation of a voluntary movement. Cereb Cortex 15:588-593.

Ferbert A, Priori A, Rothwell JC, Day BL, Colebatch JG, Marsden CD (1992) Interhemispheric inhibition of the human motor cortex. J Physiol 453:525-546.

Garry MI, Loftus A, Summers JJ (2005) Mirror mirror on the wall: viewing a mirror reflection of unilateral hand movements facilitates ipsilateral M1 excitability. Exp Brain Res 163:118-122.

Giovannelli F, Borgheresi A, Balestrieri F, Zaccara G, Viggiano MP, Cincotta M, Ziemann U (2009) Modulation of interhemispheric inhibition by volitional motor activity: an ipsilateral silent period study. J Physiol 587:5393-5410.

Godschalk M, Lemon RN, Kuypers HG, van der Steen J (1985) The involvement of monkey premotor cortex neurones in preparation of visually cued arm movements. Behav Brain Res 18:143-157.

Houzel JC, Milleret C (1999) Visual inter-hemispheric processing: constraints and potentialities set by axonal morphology. J Physiol Paris 93:271-284

Jung P, Ziemann U (2006) Differences of the ipsilateral silent period in small hand muscles. Muscle Nerve 34:431-436.

Keppel G (1991) Design and analysis: a researcher's handbook. Englewood Cliffs, NJ: Prentice Hall.

Liuzzi G, Hörniss V, Hoppe J, Heise K, Zimerman M, Gerloff C, Hummel FC (2010) Distinct temporospatial interhemispheric interactions in the human primary and premotor cortex during movement preparation. Cereb Cortex 20:1323-1331.

Loftus G, Masson M (1994) Using confidence intervals in within-subject designs. Psychon Bull Rev 1:476-490.

Mayston MJ, Harrison LM, Stephens JA (1999) A neurophysiological study of mirror movements in adults and children. Ann Neurol 45:583-594.

Meyer BU, Röricht S, Gräfin von Einsiedel H, Kruggel F, Weindl A (1995) Inhibitory and excitatory interhemispheric transfers between motor cortical areas in normal humans and patients with abnormalities of the corpus callosum. Brain 118:429-440.

Meyer BU, Röricht S, Woiciechowsky C (1998) Topography of fibers in the human corpus callosum mediating interhemispheric inhibition between the motor cortices. Ann Neurol 43:360-369.

Münchau A, Bloem BR, Irlbacher K, Trimble MR, Rothwell JC (2002) Functional connectivity of human premotor and motor cortex explored with repetitive transcranial magnetic stimulation. J Neurosci 22:554-561.

Myers JL (1979) Fundamentals of experimental design. Boston: Allyn \& Bacon.

Post M, Bakels R, Zijdewind I (2009) Inadvertent contralateral activity during a sustained unilateral contraction reflects the direction of target movement. J Neurosci 29:6353-6357.

Rokni U, Steinberg O, Vaadia E, Sompolinsky H (2003) Cortical representation of bimanual movements. J Neurosci 23:11577-11586.

Sherrington C (1909) Reciprocal innervation of antagonistic muscles. Fourteenth note- on double reciprocal innervation. Proc R Soc Lond Biol $81: 249-268$.

Smith AM (1981) The coactivation of antagonist muscles. Can J Physiol Pharm 59:733-747.

Sohn YH, Jung HY, Kaelin-Lang A, Hallett M (2003) Excitability of the ipsilateral motor cortex during phasic voluntary hand movement. Exp Brain Res 148:176-185.

Swinnen SP (2002) Intermanual coordination: from behavioural principles to neural-network interactions. Nat Rev Neurosci 3:348-359.

Teasdale N, Bard C, Fleury M, Young DE, Proteau L (1993) Determining movement onsets from temporal series. J Mot Behav 25:97-106.

Wassermann EM, Fuhr P, Cohen LG, Hallett M (1991) Effects of transcranial magnetic stimulation on ipsilateral muscles. Neurology 41:17951799.

Wise SP, Boussaoud D, Johnson PB, Caminiti R (1997) Premotor and parietal cortex: corticocortical connectivity and combinatorial computations. Annu Rev Neurosci 20:25-42. 